\title{
Bilateral Cryptorchidism, a rare presentation for persistent Müllerian duct syndrome
}

\author{
Abdullah Al-Faris ${ }^{1}$, Mosleh Jabari² ${ }^{2}$ Mohammed Al-Sayed ${ }^{1}$, Hassan Al-Shehri ${ }^{2}$
}

\author{
${ }^{1}$ Department of Pediatrics, Security Forces Hospital, Riyadh, Saudi Arabia \\ ${ }^{2}$ Department of Pediatrics, College of Medicine, Al-Imam Muhammad Ibn Saud Islamic University, Riyadh, Saudi \\ Arabia
}

Type of article: Case report

\begin{abstract}
Persistent Müllerian duct syndrome (PMDS) is a rare, sex-limited, autosomal recessive disorder representing male pseudo-hermaphroditism. It is observed in males with the presence of female reproductive organs such as the uterus, cervix, and bilateral fallopian tubes along with normally developed male reproductive organs. It generally occurs during embryogenesis due to mutation in anti-Müllerian hormone (AMH) gene, i.e., AMH gene or AMH receptor (AMHR2) gene. The present case reports a male infant with inflammation in the right groin who was admitted to Security Forces Hospital in 2015. During surgery, his testis was pulled down to the scrotum in the normal anatomical position, but full orchidopexy was not performed due to fragile capsule and edematous area. After a year, a right orchidopexy was performed. During left testis laparoscopic exploration, a small left intra-abdominal gonad in a position similar to the ovary, Müllerian structures in the form of a small uterus and vagina in the midline were observed. Biopsy of left gonad showed immature testicular tissues. The MRI findings of the pelvis showed normal male urethra with the presence of a vagina and small uterus, but no definite ovaries or testicle. Based on the clinical, laboratory, imaging, and primarily operative findings, the boy was diagnosed with PMDS. This was confirmed using genetic testing, which revealed biallelic mutations in the AMHR2 gene. The patient is currently under clinical observation to decide further management of PMDS through left testis orchidopexy, either with or without surgical excision of persistent Müllerian duct structures. The key message, which needs to be spread from this case report, is that the infant with bilateral undescended testes or inguinal hernia on one side and cryptorchidism on the other side should be thoroughly investigated to exclude any underlying disorder of sex development (DSD) before any further intervention.
\end{abstract}

Keywords: Anti-Müllerian hormone; disorder of sex development; orchidopexy; laparoscopy

\section{Introduction}

Persistent Müllerian duct syndrome (PMDS) is a rare genetic disorder representing male pseudo-hermaphroditism. It is a sex-limited autosomal recessive disorder where a male (46 chromosomes; XY) with normal external reproductive organs is characterized by the presence of female reproductive organs, namely, uterus, cervix, and bilateral fallopian tubes. Besides, he bears undescended testes, either unilateral or bilateral, as well as an upper vagina, which drains into a prostatic utricle. These female reproductive organs developed during fetal development (embryogenesis) from the Müllerian duct. A mutation observed in genes associated with anti-Müllerian hormone $(\mathrm{AMH})$, i.e., AMH gene or AMH receptor, Type II (AMHR2) gene is considered to be responsible for PMDS (1). Being a type of disorder of sex development (DSD), it is a clinical disease that requires thorough diagnosis and comprehensive treatment (2). PMDS is generally diagnosed after observing Müllerian tissue during inguinal herniorrhaphy or orchidopexy. However, very few studies report the diagnosis of PMDS using laparoscopy (3). We report a case with PMDS, which was diagnosed during laparoscopic exploration.

\section{Corresponding author:}

Assistant professor Dr. Hassan Al-Shehri, Department of Pediatrics, College of Medicine, Al-Imam Muhammad Ibn Saud Islamic University, P.O Box: 7544, Riyadh 13317-4233, Riyadh, Kingdom of Saudi Arabia, E-mail: dr.h.alshehri@hotmail.com

Received: August 09, 2016, Accepted: October 10, 2016, Published: December 2016

iThenticate screening: October 10, 2016, English editing: November 14, 2016, Quality control: December 02, 2016

(C) 2016 The Authors. This is an open access article under the terms of the Creative Commons Attribution-NonCommercialNoDerivs License, which permits use and distribution in any medium, provided the original work is properly cited, the use is non-commercial and no modifications or adaptations are made. 


\section{Case presentation}

A 14-month-old Saudi boy was admitted to the Security Forces Hospital in 2015. He was at the age of 27 days with swelling in the right groin area. Patient's medical history revealed that he was born following full-term pregnancy and normal spontaneous vaginal delivery. He had a smooth perinatal course with a birth weight of $3.5 \mathrm{~kg}$. No history of abdominal distention, greenish vomiting, or constipation was reported. The baby had optimal feeding and activity. On examination, the baby looked healthy and physically fit with no obvious dysmorphic features. His vital signs and growth parameters were normal for his age. However, a swelling of around $1 \mathrm{~cm} \times 1.5 \mathrm{~cm}$ was observed in the right groin, which was irreducible to the inguinal canal. Redness on the skin of the right groin area was also observed, which extended to the right scrotum. After examination, the patient was admitted to pediatric surgery service where he was recommended to undergo surgery. Under general anesthesia and skillful surgical management, his right testis with epididymis was found edematous and bruised with hemorrhagic areas in the right inguinal canal, but was completely viable. No clear twist was identified in the spermatic cord structures. The testis was pulled down to the scrotum in the normal anatomical position. As performing full orchidopexy (also known as orchidopexy) was not possible due to fragile capsule and edematous area, the surgeon did not continue the surgery. Left testis was not palpable in the scrotum or in the groin area. The patient was followed up after a year. He was again admitted to the hospital to undergo right orchidopexy and left testis exploration. The right testicle orchidopexy was successfully performed, whereas laparoscopic exploration in the left side revealed small left intra-abdominal gonads in a position similar to ovary, Müllerian structures in the form of a small uterus and vagina in the midline. A biopsy was performed from the left gonad, and the procedure was terminated until further assessment by a pediatric endocrinologist. The results of biopsy and histology of tissue showed immature testicular tissues. The pediatric endocrinology team was consulted to rule out any possibility of disorders of sex development (DSD) based on the intraoperative findings. The assessment of endocrine service revealed that the infant was thriving with normal vital signs and no hyper-pigmentation. Genital examination revealed a normal scrotum, but the left testis was not palpable in the scrotum or inguinal canal. Findings of laboratory examination are presented in Table 1. The magnetic resonance imaging (MRI) findings of the pelvis showed normal male urethra with the presence of a vagina and small uterus but no definite ovaries or testicle. There was a small oval cystic structure in the left inguinal canal measuring about $1.7 \times 1.4 \times 0.7 \mathrm{~cm}$. The right testicle seen in the right scrotum measured about $2 \times 1.2 \times 1 \mathrm{~cm}$. Based on the clinical, laboratory, imaging, and primarily operative findings, the boy was diagnosed with PMDS. To confirm the same, genetic testing to determine the mutation in AMH or AMHR genes was conducted and revealed biallelic mutations in AMHR2 gene (Table 2). The patient currently is undergoing close clinical follow-up by pediatric endocrinology department so as to decide performing left testis orchidopexy, either with or without surgical excision of persistent Müllerian duct structures.

Table 1. Findings of laboratory examination

\begin{tabular}{|l|l|l|}
\hline Test parameters & Result & Reference range \\
\hline Sodium* & 136 & $136-145$ \\
\hline Potassium* & 3.9 & $3.3-5.1$ \\
\hline Chloride* $^{*}$ & 103 & $98-106$ \\
\hline Bicarbonate* & 22 & $22-29$ \\
\hline Urea* $^{*}$ & 2.9 & $4.2-8.3$ \\
\hline Creatinine $^{\wedge}$ & 21 & $21-36$ \\
\hline Random blood glucose* & 5.7 & $4.4-11.1$ \\
\hline Magnesium* & 0.89 & $0.70-1.07$ \\
\hline Calcium* $^{*}$ & 2.52 & $2.1-2.6$ \\
\hline Phosphorus & 1.51 & $1.1-2$ \\
\hline 17-hydroxyprogesterone\# & 0.10 & $0.1-1.3$ \\
\hline 11-Deoxycorticosterone\$ & 23 & $43-160$ \\
\hline Anti- Müllerian Hormone\# & 137 & $7.4-243$ \\
\hline ACTH\$ & 5.42 & $7.2-63.3$ \\
\hline
\end{tabular}

*: $\mathrm{mmol} / \mathrm{l} ; \#: \mathrm{ng} / \mathrm{ml} ;{ }^{\wedge}: \mu \mathrm{mol} / \mathrm{l} ; \$: \mathrm{pg} / \mathrm{ml}$

Table 2: Results of genetic testing to determine mutation in anti-Müllerian hormone related genes

\begin{tabular}{|l|l|l|l|}
\hline Gene & Variant & Zygosity & Mode of Inheritance \\
\hline AMHR2 & c.994C $>$ T & Homozygous & Autosomal Recessive \\
NM_020547.2 & p.Arg332 & & \\
\hline
\end{tabular}




\section{Discussion and conclusions}

PMDS is a rare genetic disorder in normal males, which is described by the presence of Müllerian duct derivatives. It was first observed and explained by Nilson in 1939 (4). Though 0-1 births are observed with PMDS per 100,000 live births, about 150 cases have already been reported with $\operatorname{PMDS}(4,5)$. It is generally diagnosed using various imaging techniques such as ultrasonography, computerized tomography, MRI, etc. (4). Though these imaging techniques are useful in diagnosis of PMDS, its pre-operative diagnosis is rarely performed due to the normal development of penis and scrotum. As the patients with PMDS are generally observed with bilateral undescended testes or inguinal hernia on one side and cryptorchidism on the other side, the disorder is usually diagnosed during surgery for inguinal hernia or bilateral undescended testes (6). In the present case report, the child was diagnosed during laparoscopic exploration, but after performing right testicle orchidopexy. The fluctuation in serum AMH levels might also prove to be helpful in determining PMDS in case of infants and young boys (before puberty) (6). Studies report that patients with AMH gene defects are diagnosed with low AMH levels from birth. However, the cases with high AMH levels suffer from AMHR gene mutations attributing to insensitivity of the target tissues. AMH level is measurable before puberty, as, after pubertal age, these levels cannot be used to determine the gene responsible for PMDS in patients $(7,8)$. Genetic examination of PMDS is found to be a useful tool for its diagnosis. It included analysis of AMH and AMHR2 gene for mutation. It has been reported that mutations in AMH or AMHR2 genes are observed in almost $85 \%$ of PMDS cases, whereas in rest of $15 \%$ cases, the cause is unknown (7). In the present study, a homozygous change, i.e., NM_020547.2 demonstrated a biallelic mutation in AMHR2 gene, which is associated with PMDS. Such an alteration (p.Arg332) resulted in a premature STOP codon at amino acid position 332 and led to a truncated protein. As per American College of Medical Genetics and Genomics (ACMG) guidelines, the variant (p.Arg332) is classified as pathogenic mutation in AMHR2 gene. The management of PMDS includes complete surgical excision. Orchidopexy also can be performed in cases of children. In case of adults, orchidectomy is followed by testosterone supplementation (4). The limitation of the present study is that the pediatricians could not diagnose PMDS prior to surgery and have not been able to yet manage it properly. A key message from this case report is that, in an infant with bilateral undescended testes or inguinal hernia on one side and cryptorchidism on the other side, a thorough investigation is warranted to exclude an underlying DSD such as PMDS before any further intervention. The standard management of those patients should include testicular histology, orchidopexy, and excision of Müllerian duct structures as the risk of malignancy is higher.

\section{Acknowledgments:}

The authors are thankful for Security Forces Hospital (Riyadh, Kingdom of Saudi Arabia) for supporting this study.

\section{Conflict of Interest and authors' contributions:}

There is no conflict of interest to be declared. All authors contributed to this project and article equally. All authors read and approved the final manuscript.

\section{References:}

1) Vandersteen DR, Chaumeton AK, Ireland K, Tank ES. Surgical management of persistent müllerian duct syndrome. Urology. 1997; 49(6): 941-5. doi: 10.1016/S0090-4295(97)00104-0. PMID: 9187705.

2) Ocal G. Current concepts in disorders of sexual development. J Clin Res Pediatr Endocrinol. 2011; 3(3): 105-14. doi: 10.4274/jcrpe.v3i3.22. PMID: 21911322, PMCID: PMC3184510.

3) Kaya M, Ozcakir E, Aydiner C. Persistent mullerian duct syndrome in a child: case report and review of literature. Pediatric Urology Case Reports. 2014; 1(1): 10-5. doi: 10.14534/PUCR.201414116.

4) Madhavi D. Persistent Mullerian Duct Syndrome-A Case Report. Int J Biomed Res. 2013; 4: 124-6. doi: 10.7439/ijbr.v4i2.771.

5) Di Clemente N, Belville C. Anti-Müllerian hormone receptor defect. Best Pract Res Clin Endocrinol Metab. 2006; 20(4): 599-610. doi: 10.1016/j.beem.2006.09.004. PMID: 17161334.

6) Ozturk H, Eroglu M, Ozturk H, Uzunlar AK, Okur H. Persistent Müllerian duct syndrome associated with transverse testicular ectopia: report of two cases. Fetal Pediatr Pathol. 2007; 26(1): 41-6. doi: 10.1080/15513810701394660. PMID: 17613045.

7) Nishi MY, Domenice S, Maciel-Guerra AT, Zaba Neto A, Silva MA, Costa EM, et al. Analysis of antiMüllerian hormone (AMH) and its receptor (AMHR2) genes in patients with persistent Müllerian duct syndrome. Arq Bras Endocrinol Metabol. 2012; 56(8): 473-8. doi: 10.1590/S0004-27302012000800002. PMID: 23295284.

8) Mohammadi Sichani M, Heidarpour M, Dadkhah A, Rezvani M. Persistent mullerian duct syndrome with an irreducible inguinal hernia. Urol J. 2009; 6(4): 298-300. 